\title{
Intestinal Ischaemia Associated with Carcinoid Tumor: A Case Report with Review of the Pathogenesis
}

\section{Yener $\mathbf{O}$.}

Department of Surgery, Göztepe Training and Research Hospital, Istanbul, Turkey

Received May 3, 2012; Accepted January 15, 2013.

Key words: Carcinoid tumor - Intestinal ischaemia

Abstract: Carcinoid tumors are rare, slow-growing neuroendocrine neoplasms that are often indolent and may not become clinically apparent until there is a metastatic spread or evidence of carcinoid syndrome. A 44-year-old man presented to our clinic department with a history of previous left colon cancer operation, chronic crampy left lower quadrant pain, mass and severe anemia. A MR scan was obtained which demonstrated a calcified mesenteric mass $12 \times 8 \times 10 \mathrm{~cm}$ diameter with surrounding left colon mesenteric infiltration. The liver was normal. A case of ischaemic ileal necrosis is reported. It was associated with elastic vascular sclerosis produced by mesenteric metastases of an ileal carcinoid tumor. It is postulated that intestinal ischaemia may be of more importance in the production of abdominal pain by carcinoid tumors than has been generally accepted, and that it is the result of functional and structural changes in and around the mesenteric blood vessels, caused by substances secreted by the carcinoid tumor.

Mailing Address: Dr. Oktay Yener, Department of Surgery, Göztepe Training and Research Hospital, Plaj yolu yıldız sok. Kaya apt. No:16 D:10 caddebostan, Istanbul, Turkey; Mobile Phone: 905337088 469; e-mail: oktayener@gmail.com 


\section{Introduction}

Tumors of the neuroendocrine system account for about $2 \%$ of all cancers and can be classified as either pancreatic endocrine tumors or carcinoid tumors. Approximately 12,000 cases of carcinoid tumor are diagnosed a year (Creutzfeldt and Stöckmann, 1987). They most commonly arise in the gastrointestinal system (esophagus, stomach, intestines) and are thought to regulate digestion by controlling digestive enzyme release and intestinal motility (Kothari and Mangla, 1981). About 30\% of carcinoid tumors arise in the lungs. Because carcinoid tumors originate from hormone producing tissues, many of the side effects of carcinoid tumors can be linked to the inappropriate release of various compounds into the blood stream (Kowlessar, 1989). Carcinoid tumors occur slightly more frequently in women than men with an overall rate of approximately 2.5 cases per 100,000 people. Carcinoid tumor prevalence appears to be slightly higher in African Americans with 4.48 and 3.98 cases per 100,000 African American men and women, respectively. It is diagnosed most commonly between the ages of 50 to 70. A carcinoid tumor starts in the hormone-producing cells of various organs, primarily the gastrointestinal tract (such as the stomach and intestines) and lungs, but also the pancreas, testicles (in males) or ovaries (in females). Multiple carcinoid tumors can occur within the same organ. The cause of a carcinoid tumor is unknown (Modlin and Sandor, 1997; Neary et al., 1997).

A carcinoid tumor is classified as a neuroendocrine tumor which means it starts in cells of the neuroendocrine system, the cells that produce hormones. Carcinoid tumors can produce high levels of neuropeptides and amines (hormonelike substances); however, they may not be released in high enough quantities to cause symptoms or these may be defective and, therefore, not produce symptoms. A carcinoid tumor can grow slowly for many years without causing symptoms. Although this tumor is malignant (cancerous), a carcinoid tumor is often referred to as "cancer in slow motion" (Anthony and Drury, 1970; Brada et al., 1997; Frank et al., 1999).

\section{Case report}

A 44-years-old man was admitted to emergency clinic with malaise, palpitation, left lower quadrant abdominal pain, and constipation. The patient's medical history revealed a left colectomy due to a left colon cancer 7 years before. The patient had a strong family history of colon cancer. Physical examination of the abdomen revealed palpable mass and tenderness in the left lower quadrant. His complete blood examination was normal except severe anemia. A magnetic resonance of the abdomen and pelvis revealed a solid mass $12 \times 10 \times 8 \mathrm{~cm}$ diameter in the terminal ileum surrounded by transverse colon mesentery (Figure 1). The patient underwent a laparotomy. The tumor was located in the ileum, $8-10 \mathrm{~cm}$ from the ligamentum Treitz (Figure 2). The tumor was surrounded by transverse colon. A partial small bowel resection was performed with complete resection of the tumor, followed by 

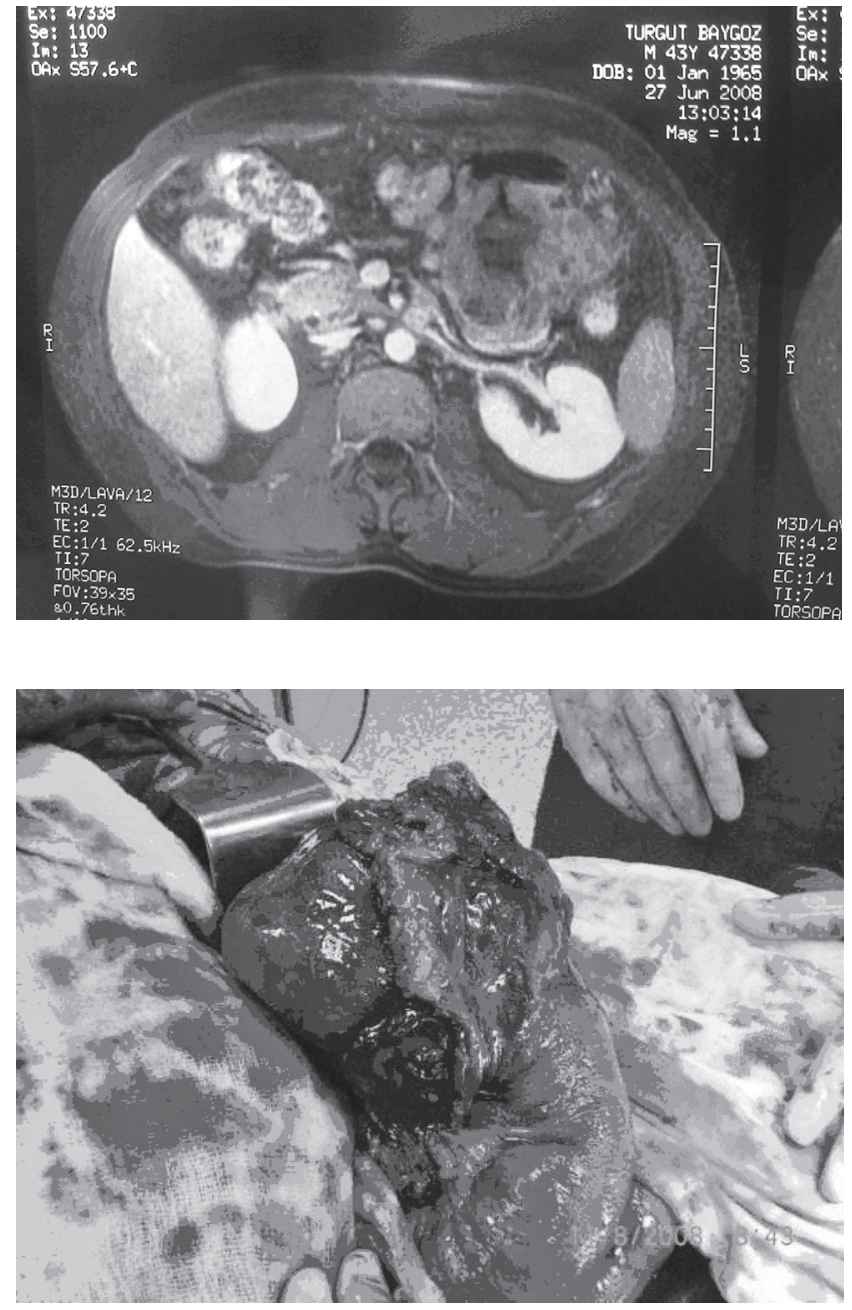

Figure 1 - A magnetic resonance of the abdomen and pelvis revealed a solid mass $12 \times 10 \times 8$ diameter in the terminal ileum surrounded by transverse colon mesentery.

Figure 2 - Peroperative explorative findings. The tumor was located in the ileum, $8-10 \mathrm{~cm}$ from the ligamentum Treitz. The tumor was surrounded by transverse colon.

primary side to side anastomosis. Tumor resection and primary repair of the serosa in the left colon was performed.

Progressive abdominal distension, rebound tenderness and general condition worsening was developed postoperatively. A free fluid in the abdomen was shown on CT scan. The patient was re-operated for acute abdomen on third day. Exploration findings shown general small bowel necrosis starting 5-6 cm from the ligamentum Treitz to the 7-8 cm ileocaecal valve. It was due to direct vascular involvement of the tumor. Previous small bowel anastomosis was normal. Resection of the effected small bowel was performed. lleojejunal anastomosis and caecostomy was done. Draine was inserted to the douglas and around anastomosis area. Histologic examination revealed a large carcinoid tumor in the ileum with metastasis to regional lymph nodes. Focal areas of mucosal ulceration, as well as 
extensive perineural and vascular invasion were present. A gastrointestinal small bowel fistula developed postoperatively within sixth days. High-output small bowel fistula was treated by Total Parenteral Nutrition. The patient was discharged on the $75^{\text {th }}$ day without any complaint. The patient consulted to the gastroenterology clinic for short bowel syndrome.

\section{Discussion}

Endocrine tumors of the digestive system are rare events and present with widely variable and often dramatic clinical syndromes. Several synonyms are currently used by clinicians and pathologists for these tumors, including "carcinoid tumor", “APUD-oma”, "gastro-entero-pancreatic (GEP) tumor”, “islet cell tumor”, "neuroendocrine tumor", and "neuroendocrine carcinoma". The term "carcinoid" was introduced in 1902 by Oberndorfer for malignant tumors arising in the small intestine that formed metastases but differed from other malignancies by a slow tumor growth (Oberndorfer, 1907).

Professor G. Rindi, Chief of the Department of Pathology, University of Brescia, Italy, introduced a discussion on the classification of neuroendocrine tumors based on proceedings of the recent World Health Organization conference, which also included other leading pathologists (Solcia et al., 2000). According to this classification scheme, neuroendocrine tumors should be stratified into well-differentiated neuroendocrine tumors and poorly differentiated small-cell neuroendocrine carcinoma (Polak, 1993).

Malignant tumors of the small intestine are rare and their symptoms are discrete and of a chronic nature. At times they manifest as an acute abdomen, with high mortality. Carcinoid tumors represent one of the types of intestinal tumors, of the neuroendocrine line, more frequently located in the cecal appendix. In the small intestine, carcinoid tumors are most frequently located in the ileum. Carcinoid tumors produce several hormonal substances, some of which may induce symptoms such as the carcinoid syndrome. Gangrene of the small intestine is a rare complication attributed to hormonal substances produced by the tumor which causes high mortality rates. Carcinoid of the midgut has an incidence between 0.46 and 1.13 per 100,000 persons per year.

Moertel in 1961 was one of the first to report bowel ischemia in four carcinoid patients (Moertel et al., 1961). Ten years later Anthony and Drury (1970) described elastic vascular sclerosis as a morphologic substrate for this disorder in carcinoid patients. Due to its rare occurrence intestinal ischemia at diagnosis and during the course of the disease is not always considered as the main cause of abdominal pain in these patients. Mesenteric fibrosis, nodular involvement, progressive tumor growth and peritoneal adhesions causing intermittent subileus are other well known complicating factors.

Mesenteric ischemia associated with carcinoid tumors often presents with nonspecific abdominal pain and is usually due to mesenteric branch artery 
occlusion caused by elastic vascular sclerosis. Mesenteric ischemia was defined by the operative findings of cyanosis or infarction.

We conclude that in patients with midgut carcinoid tumors, the histologically proved narrowing and occlusion of peripheral mesenteric arteries most likely represents elastic vascular sclerosis. It indicates mesenteric invasions of tumor, and poorly correlates with the presence of ischemia in the subtended bowel.

\section{References}

Anthony, P. P., Drury, R. A. (1970) Elastic vascular sclerosis of mesenteric blood vessels in argentaffin carcinoma. J. Clin. Pathol. 23, 110-118.

Brada, S. J., Wijffels, R. T., Kahraman, T., de Vries, E. G. (1997) Sublingual nitrate provides cause for fear of food in a carcinoid patient. Ann. Oncol. 8, 1053-1054.

Creutzfeldt, W., Stöckmann, F. (1987) Carcinoids and carcinoid syndrome. Am. J. Med. 82, 4-16.

Frank, M., Klose, K. J., Wied, M., Ishaque, N., Schade-Brittinger, C., Arnold, R. (1999) Combination therapy with octreotide and alpha-interferon: effect on tumor growth in metastatic endocrine gastroenteropancreatic tumors. Am. J. Gastroenterol. 94, 1381-1387.

Kothari, T., Mangla, J. C. (1981) Malignant tumors associated with carcinoid tumors of the gastrointestinal tract. J. Clin. Gastroenterol. 3, 43-46 (Suppl. 1).

Kowlessar, O. D. (1989) The carcinoid syndrome. In: Gastrointestinal Disease, $4^{\text {th }}$ Ed., eds. Sleisenger, M. H., Fordtran, J. S., Pp. 1560-1570, WB Saunders, Philadelphia.

Modlin, I. M., Sandor, A. (1997) An analysis of 8305 cases of carcinoid tumors. Cancer 79, 813-829.

Moertel, C. G., Sauer, W. G., Dockerty, M. B., Baggenstoss, A. H. (1961) Life history of the carcinoid in the small intestine. Cancer 14, 901-912.

Neary, P. C., Redmond, P. H., Houghton, T., Watson, G. R., Bouchier-Hayes, D. (1997) Carcinoid disease: review of the literature. Dis. Colon Rectum 40, 349-362.

Oberndorfer, S. (1907) Karzinoide Tumoren des Dünndarms. Frankf. Z. Pathol. 1, 426-429.

Polak, J. M. (1993) Diagnostic Histopathology of Neuroendocrine Tumours. Churchill Livingstone, Edinburgh.

Solcia, E., Klöppel, G., Sobin, L. H. (2000) Histological typing of endocrine tumours. In: World Health

Organization International Histological Classification of Tumours, $2^{\text {nd }}$ Ed. Springer, Berlin, New York. 